\section{BNA Yields Widely Tunable THz Source}

The terahertz $(\mathrm{THz})$ region has attracted much attention in recent years for its numerous potential spectroscopic applications in astronomy, earth science, biology, and medicine, as well as applications in communications and national security. However, in most cases, $\mathrm{THz}$ sources are limited in their tunability requiring a relative large number of different source systems when trying to cover a wide frequency range. Only organic nonlinear optical (NLO) materials have shown promise for wide tunability.

K. Miyamoto and H. Minamide at RIKEN Sendai, M. Fujiwera and H. Hashimoto at Osaka City University, and H. Ito at RIKEN Sendai and Tohoku University, Sendai, have demonstrated a widely tunable $\mathrm{THz}$-wave generation system using difference-frequency generation (DFG) in N-benzyl- 2-methy 1-4-nitroaniline (BNA) which has a large tuning capability from $0.1 \mathrm{THz}$ to $15 \mathrm{THz}$ and the highest NLO coefficient $\mathrm{d} 33$ for any yellow NLO material with $234 \mathrm{pm} / \mathrm{V}$.

As described in the February 1 issue of Optics Letters (p. 252), researchers at RIKEN Sendai, Japan prepared large BNA crystals $(\varphi 8 \times 30 \mathrm{~mm})$ using the vertical Bridgman method. BNA is an N-derivative of 2-methyl-4-nitroaniline (MNA). BNA was developed to replace MNA because of the difficulty in growing large crystals of MNA due to its unfavorable morphology and thermal decomposition behavior as well as its hygroscopicity. With the development of BNA, the researchers were able to eliminate these drawbacks. In addition, BNA exhibits large second-order optical nonlinearity and low refractive index dispersion between the optical and the $\mathrm{THz}$ region.

The researchers excited DFG in BNA using a double-crystal $\mathrm{KTiOPO}_{4}(\mathrm{KTP})$ optical parametric oscillator (OPO) pumped by a frequency-doubled Nd:YAG laser. The maximum energy was measured at $1.1 \mathrm{~mJ} /$ pulse at dual wavelength $(887 / 902 \mathrm{~nm})$. The conversion efficiency was nearly $11 \%$, corresponding to a quantum efficiency of $19 \%$ with a peak power of $160 \mathrm{~kW}$ and an oscillation threshold of $2.5 \mathrm{~mJ} /$ pulse $\left(\sim 5 \mathrm{MW} / \mathrm{cm}^{2}\right)$.

By controlling the KTP OPO at high speed, the BNA can jump to any $\mathrm{THz}$ frequency without scanning through the intermediate wavelengths and at the same time permits continuous-frequency scanning in the $0.1-15 \mathrm{THz}$ range by mixing the output of the KTP OPO over 860-902 $\mathrm{nm}$. The researchers said that an ultrawide band (0.1-40 THz) THz-wave source is achievable by combining BNA
(0.1-15 THz) and 4-dimethylamino-Nmethyl-1-4-stilbazolium-tosylate (DAST) (which the researchers had previously shown to be tunable over the range 1.5-40 THz) organic nonlinear optical crystals.

Alfred A. ZINN

\section{Microstructured Monolithic Dielectric Surface Provides High Optical Reflectivity and Low Mechanical Loss}

Optomechanical systems have attracted more and more research interest in many fields of physics. The interface between the light field and the solid-state matter needs to provide high reflectivity and low mechanical losses to enable applications such as laser cooling of mechanical oscillators, optical traps for mirrors, generation of entangled test masses, quantum nondemolition interferometry, and gravitational wave detection. However, current approaches using multilayer dielectric coatings attached to monocrystalline materials cannot realize high optical and mechanical quality simultaneously. F. Brückner of Friedrich Schiller University Jena, O. Burmeister of Leibniz University Hannover, and their colleagues have found that a microstructured monolithic dielectric surface with a subwavelength T-shaped grating profile can theoretically provide $100 \%$ reflectivity and lowest mechanical loss.

As reported in the February 1 issue of Optics Letters (p. 264), the researchers proposed a new approach by etching T-shaped gratings into the surface of a monolithic device to replace the thick multilayer stack. The T-shaped grating structure consists of a high fill factor grating on top of a low fill factor grating, which acts as an effective media low-index layer. By utilizing the resonant behavior of light coupling, this structure can be optimized to create $100 \%$ reflectivity for particular conditions of light incident from air. This approach avoids the need to add additional material to the mirror device that would potentially increase the mechanical loss.

A crystalline silicon surface was chosen to demonstrate their theory. The calculation showed that such a T-shaped grating surface can reach $95 \%$ reflectivity for a broad wavelength range of $1550 \mathrm{~nm} \pm 175 \mathrm{~nm}$ $\left(\varphi=0^{\circ}\right)$ and an angle of incidence of $\varphi=0^{\circ}$ $\pm 23^{\circ}(\lambda=1550 \mathrm{~nm})$ for TM-polarized light. Furthermore, the reflectivity was calculated to exceed a value of $99.99 \%$ for $1.48 \mu \mathrm{m}$ $<\lambda<1.58 \mu \mathrm{m}$ and $\varphi=0^{\circ} \pm 4.5^{\circ}$, respectively. Based on these evidences, the researchers concluded that their proposed novel monolithic mirror architecture offers new routes for many fields of experimental physics to prevent mechanical as well as optical loss in optomechanical devices.

ZHAOYONG SUN

\section{Negative Refractive Index Demonstrated in 3D Metamaterials at Optical Frequencies}

The prospect of invisibility cloaking through artificially structured metamaterials with negative refractive index has received a lot of media attention. For optical frequencies, however, the principle has previously only been demonstrated in two-dimensional systems unsuitable for practical applications. In the January issue of Nature Materials (p. 31; DOI: 10.1038/ nmat2072), N. Liu and colleagues from the University of Stuttgart, Germany, have reported a procedure for creating threedimensional (3D) metamaterials with negative values of magnetic permeability $(\mu)$ and electric permittivity $(\varepsilon)$ in regions of the optical spectrum, as required for a negative index material.

The researchers built stacks of split ring resonators (SRRs), conducting nanoscale structures shaped like square horseshoes that mimic magnetism at high frequencies. They fabricated the SRRs by electron beam lithography of gold thin films, adding layers by spin coating a spacer layer of solidifiable photopolymer and repeating the gold deposition and lithography. Gold markers on the substrate enabled registry of subsequent layers and uniform stacking of SRRs. The researchers built structures up to four layers thick and measured the reflectance spectra with a Fourier transform spectrometer paired with an infrared microscope. They compared the spectral reflectance to numerical simulations and determined the physical origins of the spectral features through snapshots of the simulated current distributions in the SRRs.

Negative permittivity $\varepsilon$ occurred around $80 \mathrm{THz}$ for parallel-polarized light and corresponded to the currents in all four stacked SRRs circulating inphase. For perpendicularly polarized light, negative permeability $\mu$ occurred around $120 \mathrm{THz}$ and corresponded to an antisymmetric mode of current oscillation. The researchers interpreted this behavior using a model of plasmon hybridization, in which plasmon-like oscillations of charge density couple between adjacent SRRs, resulting in hybridized symmetric and antisymmetric modes. The antisymmetric modes that lead to negative $\mu$ are only possible due to the vertical stacking of SRRs.

The spectral features and magnitude of the effect depend on the number of stacked layers and the vertical spacing 
between SSRs, as demonstrated through simulations and measurements. The researchers conclude that by combining two SRR metamaterials, one with negative $\mu$ and the other with negative $\varepsilon$, a 3D negative index material could be achieved. In addition to the media-favorite of invisibility cloaking, such a material could find application in optical computing, nanoscale imaging, and high-speed communications.

Alison HatT

\section{Atomistic Approach Predicts Resonance Frequency of Viral Capsids for Mechanodestruction Treatment}

Viruses cause many severe diseases for humans, animals, and plants, adversely affecting public health and agricultural production. Treatment options for viral infections are typically based on drug chemotherapies or vaccines, which often lead to negative side effects. Furthermore, mutations in the virus structure may make vaccines ineffective. Recently, researchers have proposed to attack viruses not through chemical but through mechanical means, by using hypersound, T-rays, or light scattering radiation. These methods are based on the idea to excite lowfrequency mode vibrations at their resonance frequency, which leads to rupture of the viral capsid structure and thereby a destruction of the virus. A similar phenomenon is known at macroscopic scales from bridges and buildings, which may suddenly collapse if excited at their resonance frequencies by wind loads. In order to optimally apply this new treatment option, it is desirable to know the resonance frequencies of the virus structure.

How can one predict these resonance frequencies, provided the atomic structure of the virus is known? In the January 18 issue of Physical Review Letters (028101; DOI: 10.1103/PhysRevLett.100.028101), E.C. Dykeman and O.F. Sankey of Arizona State University describe a new computational method to predict the low-frequency vibrational modes of biological structures based on their atomistic geometry. The new approach is based on an energy functional from electronic structure theory and provides a much more efficient strategy to analyze the atomistic properties of virus vibrations than earlier attempts. The method is robust and is now being applied to plant viruses (e.g., cowpea chlorotic mottle virus), bacteriophages (e.g., M13 bacteriophage), and human viruses (e.g., polio and hepatitis B).

A significant advantage of Dykeman and Sankey's approach is that it allows for an all-atom description of the vibrational mode patterns based on the atomistic structure of the virus, which can be obtained from an x-ray diffraction analysis of the virus geometry.

Using the new approach, Dykeman and Sankey calculated the vibrational modes of the satellite tobacco necrosis virus based on the atomistic geometry obtained from an earlier $x$-ray diffraction analysis. Their calculations result in the prediction of the specific resonance frequencies of excitation that will most likely lead to a destruction of the virus. It also provides insight into the particular molecular deformation mechanisms, such as shearing of beta-sheets. This new tool could be an important computational tool in developing new treatment options for newly discovered types of viruses, according to the researchers.

This theoretical group works in close collaboration with an experimental effort led by colleague K.-T. Tsen of Arizona State, who has had success in inactivating viruses by inelastic light scattering. Future studies may include experimental verification of the predicted vibrational frequencies of specific viruses.

MARKUS J. BUEHLER

\section{CNT FET with Asymmetric Contact Shows Single Electron Transistor Characteristics at Low \\ Temperature}

Recent studies have shown that carbon nanotube (CNT) room-temperature single electron transistors (RTSETs) have been obtained by manipulation with an atomic force microscope (AFM) and by local chemical modification. However, almost all reported studies on RTSETs are with symmetric drain (D) and source (S) electrodes. Now, H. Li and Q. Zhang from Nanyang Technological University, Singapore and N. Marzari from the Massachusetts Institute of Technology have reported on a type of short singlewalled CNT (SWCNT)-field-effect transistor (FET), which shows coulomb blockade effects with asymmetric D and S electrodes at low temperatures. Moreover, the devices exhibit current rectification characteristics at room temperature.

In the January issue of Nano Letters (p. 64; DOI: 10.1021/nl071905e), the researchers report the fabrication of SWCNT-FETs with a channel length of $~ 90$ $\mathrm{nm}$. The researchers started with a highly boron-doped silicon wafer, which was capped by thermal oxide of $500 \mathrm{~nm}$. Their interdigital electrode of $\mathrm{Au} 90 \mathrm{~nm} / \mathrm{Ti}$ $10 \mathrm{~nm}$ thickness was patterned on this thermal oxide. SWCNTs were placed across these electrodes by a dielectrophore- sis technique. They specifically kept the concentration of the SWCNT suspension very low in order to have only a few SWCNTs bridged between these interdigital electrodes. The $\mathrm{Al}$ (source) was deposited in a way so that one end of the SWNTchannel was attached to the sidewall of the $\mathrm{Au}$ (drain) electrodes and the other end was buried beneath the deposited $\mathrm{Al}$ layer in order to create a shallow gap between the $\mathrm{Al}$ and $\mathrm{Au}$ electrodes. At room temperature, they found that the device shows diode-like characteristics with a maximum current rectification ratio of $\sim 10^{4}$. But at $25 \mathrm{~K}$, they found a diamond-shaped current suppression region in the contour plot of the drain-source current mapped in a plane as a function of the drain-source bias voltage (on one axis) and the gate-source voltage bias (on the other axis), which they identified as the Coulomb blockade effect that is usually found in single electron transistors. With increasing temperature, the thermal energy becomes comparable to or even larger than the Coulomb charging energy. As a result, the oscillation peaks broaden and fade away when the temperature is $\sim 100 \mathrm{~K}$.

The researchers concluded that due to the unequal thermionic emission current in the asymmetric contacts (as a result of their different barrier heights), they saw asymmetric current amplitudes under positive and negative drain-source voltage bias. The barrier height for electrons at the $\mathrm{Au} / \mathrm{SWCNT}$ contact is larger than that at the Al/SWCNT contact by a factor of $>2$ for all gate voltages. In an atmosphere environment, the device shows significant diode-like effects with maximum current rectification ratios of $>10000$.

M. GOWTHAM

\section{Slithering of Shear Bands Can Improve Plasticity in Bulk Metallic Glasses}

Glasses have evolved through many developments, starting from Obsidian (a natural glass) to metallic glasses and bulk metallic glasses (BMGs). BMGs have been developed since the 1990s as a new class of engineering materials, which offer an opportunity to revolutionize the field of structural materials with an excellent combination of ductility, strength, and toughness.

Failure of BMGs under extensive deformation through the formation of shear bands at room temperature has been well documented for $\mathrm{Pt}-, \mathrm{Cu}-, \mathrm{Pd}, \mathrm{Ti}-$, and $\mathrm{Zr}$ based glassy alloys. However, this disadvantage has been overcome by the recent development of unique ductile BMGs.

As described in the January issue of the 